\title{
A prominent relativistic iron line in the Seyfert 1 MCG-02-14-009
}

\author{
D. Porquet
}

Max-Plank-Institut für extraterrestrische Physik, Postfach 1312, 85741, Garching, Germany

e-mail: dporquet@mpe.mpg.de

Received 26 August 2005 / Accepted 8 November 2005

\section{ABSTRACT}

I report the discovery of a prominent broad and asymmetrical feature near $6.4 \mathrm{keV}$ in the Seyfert 1 MCG-02-14-009 $(z=0.028)$ with XMM-Newton/EPIC. The present short X-ray observation (PN net exposure time $\sim 5 \mathrm{ks}$ ) is the first one above $2 \mathrm{keV}$ for MCG-02-14-009. The feature can be explained by either a relativistic iron line around either a Schwarzschild (non-rotating) or a Kerr (rotating) black hole. If the feature is a relativistic iron line around a Schwarzschild black hole, the line energy is $6.51_{-0.12}^{+0.21} \mathrm{keV}$ with an equivalent width of $631_{-243}^{+259} \mathrm{eV}$ and that the inclination angle of the accretion disc should be less than $43^{\circ}$. A relativistically blurred photoionized disc model gives a very good spectral fit over the broad band $0.2-12 \mathrm{keV}$ energy range. The spectrum is reflection dominated and this would indicate that the primary source in MCG-02-14-009 is located very close to the black hole, where gravitational light bending effect is important (about 3-4 $R_{\mathrm{g}}$ ), and that the black hole may rapidly rotate.

Key words. galaxies: active - X-rays: galaxies - accretion discs - quasars: individual: MCG-02-14-009

\section{Introduction}

The $\mathrm{Fe} \mathrm{K} \alpha$ line complex observed in the $6-7 \mathrm{keV}$ range is an important spectral tool. Indeed the determination of the origin of the $\mathrm{Fe} \mathrm{K} \alpha$ line is the most direct signature of the inner accretion disk around black hole $(\mathrm{BH})$. With the satellite ASCA several observations of broad and asymmetrical (red wing) lines were discovered with the most famous in MCG-06-30-15 (e.g., Tanaka et al. 1995; Fabian et al. 2002). These lines are commonly interpreted as emission originated from the inner part of the accretion disk around the massive BH (e.g., Tanaka et al. 1995; Fabian et al. 1995; Nandra et al. 1997; Reynolds \& Fabian 1997, and see also the recent exhaustive reviews from Fabian et al. 2000 and Fabian \& Miniutti 2005, for more detailed information). The current generation of X-ray satellites XMM-Newton and Chandra have enlightened that broad line profiles are not so common in Active Galactic Nuclei (AGN), as previously inferred from $A S C A$ data, with the large majority of AGN showing the presence of a narrow iron line near $6.4 \mathrm{keV}$ data (e.g., Reeves 2003; Bianchi et al. 2004; Page et al. 2004; Porquet et al. 2004; Piconcelli et al. 2005). From the line characteristics (shape, energy, width, equivalent width ...) primordial information about the accretion disk (inclination, emissivity profile, the inner stable circular orbit) as well as about the BH (spin) itself can be inferred (see e.g., Fabian \& Miniutti 2005).

In this Paper I present an XMM-Newton observation of Seyfert 1 MCG-02-14-009. This is the first observation of this object above $2 \mathrm{keV}$. This observation is part of an XMM-Newton survey of 21 bright ROSAT selected AGN with low intrinsic absorption. Gallo et al. (2005) reports the global properties of this sample. Here I focus on the discovery of a prominent broad and asymmetrical feature near $6.4 \mathrm{keV}$ and I investigate the possible physical origins of this feature.

Note that all fit parameters are given in the quasar's rest frame, with values of $H_{0}=75 \mathrm{~km} \mathrm{~s}^{-1} \mathrm{Mpc}^{-1}$, and $q_{0}=0.5$ assumed throughout. The errors quoted correspond to $90 \%$ confidence ranges for one interesting parameter $\left(\Delta \chi^{2}=2.71\right)$. Abundances are those of Anders \& Grevesse (1989).

\section{XMM-Newton observation}

MCG-02-14-009 was observed by XMM-Newton on September 4, 2000 (ID 0103860701; orbit 135; exposure time $\sim 9.5 \mathrm{ks}$ ). The EPIC MOS cameras (Turner et al. 2001) were operated in the Small Window mode, while the EPIC PN camera (Strüder et al. 2001) was operated in the standard Full Frame mode. The data were re-processed and cleaned (net time exposure $\sim 5 \mathrm{ks}$ for $\mathrm{PN}$ ) using the SAS VERSION 6.1.0 (Science Analysis Software) package, using the latest CCF files concerning the PN filter transmission (EPN_FILTERTRANSX_0014.CCF), the effective area of the PN X-Ray telescope (XRT3_XAREAEF_0010.CCF), and the PN spectral response (EPN_QUANTUMEF_0016.CCF, EPN_REDIST_0010.CCF). Since pile-up effect was negligible, X-ray events corresponding to patterns 0-12 and 0-4 events (single and double pixels) were selected for MOS and PN, respectively. Only good X-ray events (with FLAG $=0$ ) were included. A low-energy cutoff was set to $200 \mathrm{eV}$. 
The source spectrum and the light curve were extracted using a circular region of diameter $30^{\prime \prime}$ centered on the source position. Background spectra were taken on the same CCD than the source (excluding possible X-ray point sources). The XSPEC V11.3 software package was used for spectral analysis of the background-subtracted spectrum using the response matrices and ancillary files derived from the SAS tasks RMFGEN and ARFGEN. Since in this work, I focused on the study of the broad feature near $6.4 \mathrm{keV}$, I used only the PN data which have a much better sensitivity above $6 \mathrm{keV}$ compared to MOS data. However, I checked that similar results between the PN and MOS data were obtained. The PN spectrum were binned to give a minimum of 20 counts per bin.

The signal to noise ratio were not sufficient for reliable RGS data analysis.

\section{Spectral analysis}

In the following, I used the updated cross-sections for X-ray absorption by the interstellar medium (TBABS in XSPEC) from Wilms et al. (2000). In all subsequent fits, I included both the Galactic column density $\left(9.31 \times 10^{20} \mathrm{~cm}^{-2}\right)$ and a possible additional absorption $\left(N_{\mathrm{H}}^{\mathrm{int}}\right)$ located at the quasar redshift.

\subsection{The broad-band spectrum}

First, a single absorbed power-law model was fitted to the broad-band $0.2-12 \mathrm{keV}$ PN spectrum, but a poor fit was obtained $\left(\chi^{2}=591.2 / 396\right)$ with $\Gamma=2.12 \pm 0.03, N_{\mathrm{H}}^{\text {int }}<$ $0.13 \times 10^{20} \mathrm{~cm}^{-2}$. An absorbed broken power-law gave a much better fit of the data $\left(\chi^{2} /\right.$ d.o.f. $\left.=465.7 / 394\right)$ with $\Gamma_{\text {soft }}=$ $3.02_{-0.24}^{+0.27}, \Gamma_{\text {hard }}=1.87 \pm 0.06, E_{\text {break }}=1.37_{-0.09}^{+0.12} \mathrm{keV}$, and $N_{\mathrm{H}}^{\text {int }}=(8.2 \pm 0.3) \times 10^{20} \mathrm{~cm}^{-2}$. The inferred X-ray luminosities are: $L(0.2-12 \mathrm{keV})=2.9 \times 10^{43} \mathrm{erg} \mathrm{s}^{-1}$, and $L(2-10 \mathrm{keV})=$ $6.2 \times 10^{42} \mathrm{erg} \mathrm{s}^{-1}$.

To characterize the hard X-ray continuum, an absorbed power-law model has been fitted over the $2-5 \mathrm{keV}$ energy range where the spectrum should be relatively unaffected by the presence of a broad soft excess, a warm absorber-emitter medium, an $\mathrm{Fe} \mathrm{K} \alpha$ emission line, and a contribution above $8 \mathrm{keV}$ from a high energy Compton reflection hump. In this energy range, the data were well fitted by a single power-law model with $\Gamma=1.97 \pm 0.16\left(\chi^{2} /\right.$ d.o.f. $\left.=92.6 / 81\right)$. This powerlaw slope was similar to the value found in Seyfert type 1 (Reynolds 1997; Brandt et al. 1997). Figure 1 displays the spectrum extrapolated over the $0.2-12 \mathrm{keV}$ broad band energy. A positive residual is seen between about $0.4-0.7 \mathrm{keV}$, and specially a large and positive asymmetrical residual is clearly seen near $6.4 \mathrm{keV}$ (in the quasar rest-frame) suggesting the presence of a Fe $\mathrm{K} \alpha$ line complex.

\subsection{The prominent broad feature near $6.4 \mathrm{keV}$}

As shown in Fig. 1, a significant deviation is seen near $6.4 \mathrm{keV}$ in the quasar frame. Here, I focused on the study of the feature near $6.4 \mathrm{keV}$, therefore I fitted only the PN spectrum above $2 \mathrm{keV}$. In the overall 2-12 keV energy band, adding a Gaussian line to a single power-law model drops the $\chi^{2}$ value by 18 with

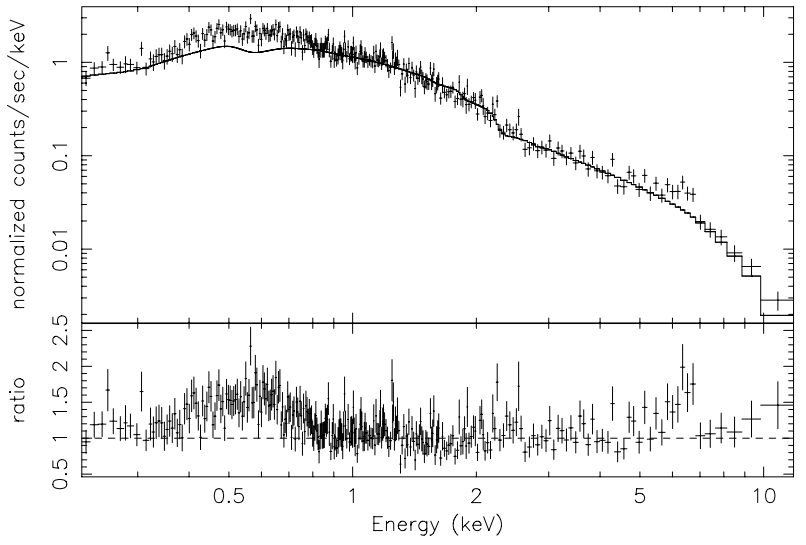

Fig. 1. The PN spectrum of MCG-02-14-009 (in the observer frame). A power-law has been fitted to the $2-5 \mathrm{keV}$ data and extrapolated to lower and higher energies. A soft X-ray positive residual is seen between about $0.4-0.7 \mathrm{keV}$, as well as a broad and significant positive deviation near $6.4 \mathrm{keV}$, suggesting the presence of a $\mathrm{Fe} \mathrm{K} \alpha$ line. For presentation, the data have been re-binned into groups of 5 bins, after group of a minimum of 20 counts per bin is used for the fit.

the addition of 3 degrees of freedom (Table 1). This line was significant at $99.8 \%$ according to the F-test. Adding an ionized emission line and an absorption edge did not improve the fit. The line was well resolved with a full width at half maximum (FWHM) velocity width of about $30000 \mathrm{~km} \mathrm{~s}^{-1}$, and a large equivalent width $(E W)$ of about $527 \mathrm{eV}$ (Table 1). The line width and the asymmetrical profile likely indicate that the X-ray emission is originating from a region close to the BH in MCG-02-14-009. The FWHM of the line was only about three times smaller than the one found in the Seyfert 1 MCG-6-30-15, which shows the most extreme broad Fe K $\alpha$ line observed up to now (e.g., $100000 \mathrm{~km} \mathrm{~s}^{-1}$, Tanaka et al. 1995; Wilms et al. 2001; Fabian et al. 2002; Lee et al. 2002). Therefore, I fitted the line with a profile expected from a relativistic accretion disk around a non-rotating (Schwarzschild) $\mathrm{BH}$, using the DISKLINE model in XSPEC from Fabian et al. (1989). I found that such a profile, with a inclination less than $43^{\circ}$ (typical for a Seyfert type 1), emitted at a rest-frame energy of about $6.5 \mathrm{keV}$, provided an excellent representation of the line observed in MCG-02-14-009 (Table 1). The line energy was not enough constrained, according to the error bars, to discriminate between a line emitted by "cold" iron (i.e. $<$ Fe XVII) or by highly ionized iron (i.e. Fe XXV). An equally good fit was obtained (Fig. 2) for a maximally rotating $\mathrm{BH}$ (Kerr) disc emission line model (LAOR; Laor 1991). A higher signal to noise ratio spectrum is required to constrain the line energy and to discriminate between the Schwarzschild and the Kerr $\mathrm{BH}$, and to determine the $\mathrm{BH}$ spin, if any. No narrow $(\sigma=10 \mathrm{eV})$ line at $6.4 \mathrm{keV}$, that could be emitted by the BLR or the molecular torus, is required by the data.

\subsection{Ionized Absorption in MCG-02-14-009?}

Alternatively, I investigated whether the strong iron $\mathrm{K} \alpha$ feature can be modeled with a warm absorber model. Indeed partial covering models where the $\mathrm{BH}$ is partially covered by an 
Table 1. Best-fitting spectral parameters of the PN spectrum (exposure time $\sim 5 \mathrm{ks}$ ) in the $2-12 \mathrm{keV}$ energy range for an absorbed (Galactic, $N_{\mathrm{H}}=9.31 \times 10^{20} \mathrm{~cm}^{-2}$ ) power-law (PL) component plus a line profile: GA: Gaussian profile; and DISKLINE (Fabian et al. 1989) and LAOR (Laor 1991): profile line emitted by a relativistic accretion disk for a non-rotating BH and a maximally rotating BH, respectively. An emissivity law $q$ equal to -2 was assumed. (a): $R_{\text {in }}=6 R_{\mathrm{g}}$ and $R_{\text {out }}=1000 R_{\mathrm{g}}$. (b): $R_{\mathrm{in}}=1.23 R_{\mathrm{g}}$ and $R_{\text {out }}=400 R_{\mathrm{g}}$. The F-test probability was estimated comparing to the single power-law model (PL).

\begin{tabular}{lccccccc}
\hline \hline Model & $\Gamma$ & \multicolumn{3}{c}{ Line parameters } & \multirow{2}{*}{$\chi^{2}$ d.o.f. } & F-test \\
& & $E(\mathrm{keV})$ & $\sigma(\mathrm{keV})$ & $\theta(\mathrm{deg})$ & $E W(\mathrm{eV})$ & & \\
\hline $\mathrm{PL}$ & $1.83 \pm 0.09$ & - & - & - & - & $145.2 / 114$ & - \\
$\mathrm{PL}+\mathrm{GA}$ & $1.92 \pm 0.11$ & $6.51_{-0.20}^{+0.16}$ & $0.28_{-0.17}^{+0.19}$ & - & $527_{-248}^{+277}$ & $127.1 / 111$ & $99.80 \%$ \\
$\mathrm{PL}+\mathrm{DISKLINE}^{(a)}$ & $1.93 \pm 0.10$ & $6.51_{-0.12}^{+0.21}$ & - & $<43$ & $631_{-243}^{+259}$ & $125.5 / 111$ & $99.90 \%$ \\
$\mathrm{PL}+\mathrm{LAOR}^{(b)}$ & $1.95 \pm 0.11$ & $6.41_{-0.18}^{+0.37}$ & - & $<49$ & $765_{-338}^{+315}$ & $125.4 / 111$ & $99.90 \%$ \\
\hline
\end{tabular}

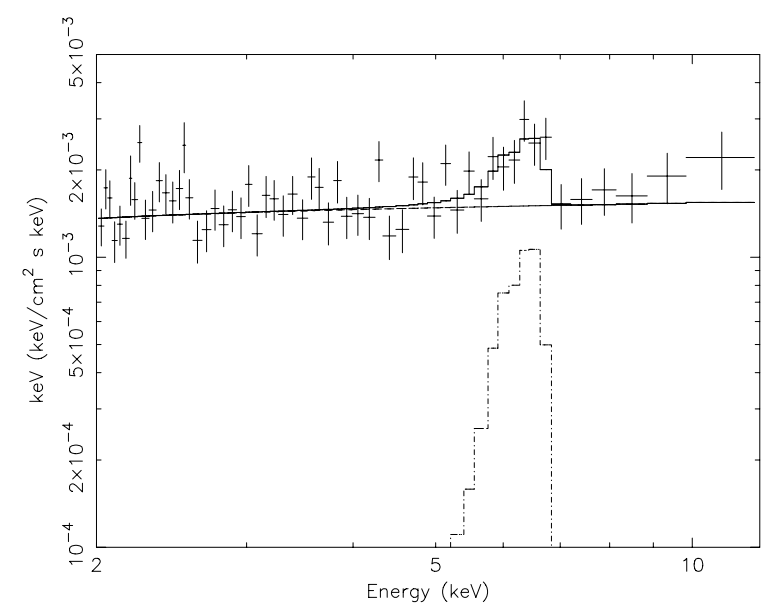

Fig. 2. The unfolded PN spectrum of MCG-02-14-009 (in the observer frame) showing the model composed of a power-law continuum and a relativistic line profile around a rotating BH (LAOR; Laor 1991). See Table 1 for the fitting parameter values.

high column density absorbing medium result in a curved continuum that could mimic a relativistic line. I used the model ABSORI in XSPEC (Done et al. 1992). The model was applied so that the X-ray power-law continuum was modified by the absorber (i.e. a partial covering). I found a reasonable fit for the continuum $\left(\chi^{2} /\right.$ d.o.f. $\left.=452.2 / 391\right)$ but there was still a broad asymmetrical residual feature near $6.4 \mathrm{keV}$. Adding a relativistic line profile (DISKLINE, assuming an emissivity law $q=-2)$, a much better fit $\left(\chi^{2} /\right.$ d.o.f. $\left.=434.0 / 390\right)$ was found with $N_{\mathrm{H}}^{\text {int }}=3.5 \pm 1.3 \times 10^{20} \mathrm{~cm}^{-2}, \Gamma=2.21 \pm 0.06$, $N_{\mathrm{H}}^{\text {absori }}=\left(7.5_{-2.3}^{+4.2}\right) \times 10^{22} \mathrm{~cm}^{-2}, \xi=244_{-57}^{+107} \mathrm{erg} \mathrm{cm} \mathrm{s}^{-1}, A_{\mathrm{Fe}}=$ $0.35_{-0.13}^{+0.17}$ (compared to solar abundance), $E_{\text {line }}=6.50_{-0.14}^{+0.22} \mathrm{keV}$, $\theta_{\text {disk }}=37_{-12}^{+14}$ degrees, and $E W_{\text {line }}=708_{-250}^{+264} \mathrm{eV}$.

\subsection{The presence of an $X$-ray ionized disc reflection?}

I tested if the whole spectrum is the signature of X-ray ionized reflection when the disk surface is irradiated with X-rays using the REFLION model in XSPEC developed by Ross \& Fabian (2005). Indeed, they have demonstrated that this model can explained in several AGN, the broad-band spectrum $(0.2-12 \mathrm{keV})$

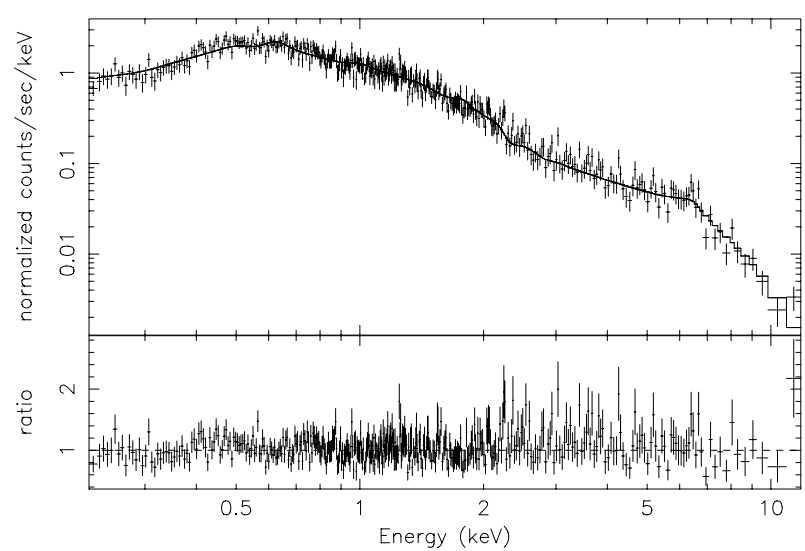

Fig. 3. The PN spectrum of MCG-02-14-009 (in the observer frame). A relativistically blurred photo-ionized disc model (REFLION; Ross \& Fabian 2005) has been fitted, and gave a good representation of the whole spectrum. A primary power-law component is taking into account in the model. See text for the fitting parameter values.

without involving several add-oc components such as blackbody, power-law continuum. For example, a signature of ionized reflection is the soft excess emission which occurs in the $0.2-2 \mathrm{keV}$ band owing to lines and bremsstrahlung from the hot surface layers. It gives a bump in the relativistically blurred spectrum which when convolved with an XMM-Newton PN response matrix mimics a blackbody of temperature $150 \mathrm{eV}$. This may explain the $100-200 \mathrm{eV}$ temperature component found in the X-ray spectra of many low-redshift PG quasars (e.g., Gierliński \& Done 2004; Porquet et al. 2004; Piconcelli et al. 2005). This model has been used successfully for example in the case of the NLS1 PG1404+226 (Crummy et al. 2005).

The PN spectrum has been fitted taking into account the relativistic effect due to relativistic motion in the inner part of the accretion disc, by blurring the spectrum with a Laor line profile. A primary power-law $(\Gamma)$ was assumed in addition to the disc reflection model. This model corresponds to the spectrum from a photoionized disc around a maximally rotating (Kerr) $\mathrm{BH}$. I let the inclination of the disc $(\theta)$ free and assumed an emissivity index of 2 and an inner radius $\left(R_{\mathrm{in}}\right)$ of $1.23 R_{\mathrm{g}}$. As shown in Fig. 3, a very good data fit is found with $\left(\chi^{2} /\right.$ d.o.f. $\left.=418.8 / 392\right)$ : $N_{\mathrm{H}}<1.1 \times 10^{20} \mathrm{~cm}^{-2}, \Gamma=1.59_{-0.23}^{+0.11}, \xi=592_{-88}^{+133} \mathrm{erg} \mathrm{cm} \mathrm{s}^{-1}$, 
$A_{\mathrm{Fe}}=0.42_{-0.29}^{+0.59}$, and $\theta<29.4^{\circ}$. This data fit is much better $\left(\Delta \chi^{2}=17.5\right.$ for only one additional free parameter) than the one found in case of a disc reflection model without taking into account the blurring effect. Letting the inner radius as a free parameter, an upper limit of about $7 R_{\mathrm{g}}$ has been found, and therefore is compatible with both a non-rotating and a rotating $\mathrm{BH}$. However, the flux contribution of the reflection component is about $76 \%$ compared to the primary power law component, this would mean that in order to produce such reflection-dominated spectrum, the primary source in MCG-02-14-009 must be very close to the $\mathrm{BH}$, where gravitational light bending effect is important, i.e. about 3-4 $R_{\mathrm{g}}$ (Miniutti \& Fabian 2004). Therefore, this would favor a rapidly rotating $\mathrm{BH}$.

\section{Conclusion}

I report here the analysis of a short XMM-Newton/EPIC observation (PN net time exposure $\sim 5 \mathrm{ks}$ ) of the Seyfert 1 MCG-02-14-009 ( $z=0.028)$. The present X-ray observation is the first X-ray observation above $2 \mathrm{keV}$ of this object. This Seyfert 1 is a weak AGN with a luminosity in the $2-10 \mathrm{keV}$ energy range of $6.2 \times 10^{42} \mathrm{erg} \mathrm{s}^{-1}$.

I discovered in the hard X-ray part of the PN spectrum a prominent broad and asymmetrical feature near $6.4 \mathrm{keV}$. I investigated the possible physical origins of this feature and I found that it can be explained by either a relativistic iron line around either a Schwarzschild (non-rotating) or a Kerr (rotating) BH. A longer X-ray follow-up with a higher signal to noise ratio spectrum is required to discriminate between these two possible origins. If the line is a relativistic iron line around a Schwarzschild $\mathrm{BH}$, the line energy is $6.51_{-0.12}^{+0.21} \mathrm{keV}$ with an equivalent width of $631_{-243}^{+259} \mathrm{eV}$ and that the inclination angle of the accretion disc should be less than $43^{\circ}$ (typical for a Seyfert type 1). While a partial covering model may explain the underlying continuum, it was unable to explain the broad feature. Interestingly the equivalent width found here is consistent with the one found for average spectrum of the X-ray background sources from a large XMM-Newton survey of the Lockman Hole field (Streblyanska et al. 2005).

A relativistically blurred photoionized disc model gives a very good spectral fit over the broad band $0.2-12 \mathrm{keV}$ energy range. The spectrum is reflection dominated and this would indicate that the primary source in MCG-02-14-009 is located very close to the $\mathrm{BH}$, where gravitational light bending effect is important (about $3-4 R_{\mathrm{g}}$ ), and that the $\mathrm{BH}$ may rapidly rotate.

The discovery of this broad and asymmetrical feature (probably relativistic iron line) is of great interest since with XMM-Newton and Chandra only few relativistic iron line have been confirmed or discovered: e.g., MCG-6-30-15 (Wilms et al. 2001), MCG-5-23-16 (Dewangan et al. 2003), Mrk 335 (Gondoin et al. 2002), and Q0056-363 (Porquet \& Reeves 2003). In conclusion, the study of such relativistic line profiles is crucial to understand the effects of strong gravity on the accreting material in the vicinity of $\mathrm{BH}$.
Acknowledgements. Based on observations obtained with the XMM-Newton, and ESA science mission with instruments and contributions directly funded by ESA member states and the USA (NASA). I would like to thank A. Fabian and J. Crummy for a helpful discussion about the relativistic blurred photoionized disc model used in this work. I thank the anonymous referee for fruitful comments.

\section{References}

Anders, E., \& Grevesse, N. 1989, Geochim. Cosmochim. Acta, 53, 197

Bianchi, S., Matt, G., Balestra, I., Guainazzi, M., \& Perola, G. C. 2004, A\&A, 422, 65

Brandt, W. N., Mathur, S., \& Elvis, M. 1997, MNRAS, 285, L25

Crummy, J., Fabian, A. C., Brandt, W. N., \& Boller, T. 2005, MNRAS, 361,1197

Dewangan, G. C., Griffiths, R. E., \& Schurch, N. J. 2003, ApJ, 592, 52

Done, C., Mulchaey, J. S., Mushotzky, R. F., \& Arnaud, K. A. 1992, ApJ, 395, 275

Fabian, A. C., \& Miniutti, G. 2005, in Kerr Spacetime: Rotating Black Holes in General Relativity, in press [arXiv: astro-ph/0507409]

Fabian, A. C., Rees, M. J., Stella, L., \& White, N. E. 1989, MNRAS, 238,729

Fabian, A. C., Nandra, K., Reynolds, C. S., et al. 1995, MNRAS, 277, L11

Fabian, A. C., Iwasawa, K., Reynolds, C. S., \& Young, A. J. 2000, PASP, 112, 1145

Fabian, A. C., Vaughan, S., Nandra, K., et al. 2002, MNRAS, 335, L1

Gallo, L., Lehmann, I., Pietsch, W., et al. 2005, MNRAS, in press [arXiv:astro-ph/0510551]

Gierliński, M., \& Done, C. 2004, MNRAS, 349, L7

Gondoin, P., Orr, A., Lumb, D., \& Santos-Lleo, M. 2002, A\&A, 388, 74

Laor, A. 1991, ApJ, 376, 90

Lee, J. C., Iwasawa, K., Houck, J. C., et al. 2002, ApJ, 570, L47

Miniutti, G., \& Fabian, A. C. 2004, MNRAS, 349, 1435

Nandra, K., George, I. M., Mushotzky, R. F., Turner, T. J., \& Yaqoob, T. 1997, ApJ, 477, 602

Page, K. L., O’Brien, P. T., Reeves, J. N., \& Turner, M. J. L. 2004, MNRAS, 347, 316

Piconcelli, E., Jimenez-Bailón, E., Guainazzi, M., et al. 2005, A\&A, 432,15

Porquet, D., \& Reeves, J. N. 2003, A\&A, 408, 119

Porquet, D., Reeves, J. N., O’Brien, P., \& Brinkmann, W. 2004, A\&A, 422,85

Reeves, J. 2003, in Active Galactic Nuclei: From Central Engine to Host Galaxy, ASP Conf. Ser., 290, 35

Reynolds, C. S. 1997, MNRAS, 286, 513

Reynolds, C. S., \& Fabian, A. C. 1997, MNRAS, 290, L1

Ross, R. R., \& Fabian, A. C. 2005, MNRAS, 358, 211

Strüder, L., Briel, U., Dennerl, K., et al. 2001, A\&A, 365, L18

Streblyanska, A., Hasinger, G., Finoguenov, A., et al. 2005, A\&A, 432, 395

Tanaka, Y., Nandra, K., Fabian, A. C., et al. 1995, Nature, 375, 659

Turner, M. J. L., Abbey, A., Arnaud, M., et al. 2001, A\&A, 365, L27

Wilms, J., Allen, A., \& McCray, R. 2000, ApJ, 542, 914

Wilms, J., Reynolds, C. S., Begelman, M. C., et al. 2001, MNRAS, 328, L27 